\title{
ON CERTAIN MEANS OF TWO ARGUMENTS AND THEIR EXTENSIONS
}

\section{EDWARD NEUMAN and JÓZSEF SÁNDOR}

Received 2 August 2002

Inequalities for three means introduced by H.-J. Seiffert are obtained. Generalizations of these means, their basic properties, and inequalities satisfied by the new class of means are also included.

2000 Mathematics Subject Classification: 26D15, 26D99.

1. Introduction. Let $x$ and $y$ be positive real numbers. The logarithmic mean and the identric mean of $x$ and $y$ are defined by

$$
\begin{gathered}
L \equiv L(x, y)=\frac{x-y}{\ln x-\ln y} \quad \text { for } x \neq y, L(x, x)=x, \\
I \equiv I(x, y)=e^{-1}\left(\frac{x^{x}}{y^{y}}\right)^{1 /(x-y)} \quad \text { for } x \neq y, I(x, x)=x,
\end{gathered}
$$

respectively. The arithmetic and geometric means of $x$ and $y$ are $A \equiv A(x, y)=$ $(x+y) / 2$ and $G \equiv G(x, y)=\sqrt{x y}$, respectively.

In 1995, Seiffert [22] has introduced the following two means:

$$
\begin{gathered}
M \equiv M(x, y)=\sqrt{L\left(A^{2}, G^{2}\right)}, \\
N \equiv N(x, y)=\sqrt{I\left(A^{2}, G^{2}\right)} .
\end{gathered}
$$

The main result of [22] states that

$$
L<M<N<I
$$

$(x \neq y)$. This gives a refinement of the well-known inequality $L<I$.

Another mean introduced by Seiffert (see [20]), denoted by $P$, is defined as follows:

$$
P \equiv P(x, y)=\frac{x-y}{4 \arctan \sqrt{x / y}-\pi} \quad \text { for } x \neq y, P(x, x)=x .
$$

It is known (see [17]) that this mean interpolates the inequality for the logarithmic and identric means, that is,

$$
L<P<I \text {. }
$$


For more inequalities involving the latter mean, the interested reader is referred to $[17,20,21]$.

This paper deals, among other things, with the refinements of inequalities (1.5) and (1.7), and is organized as follows. Definitions and basic properties of other means used in this paper are given in Section 2. Refinements of (1.5) and (1.7) are established in Section 3. The last section deals with inequalities for the Seiffert means of order $t(t \in \mathbb{R})$, where $M$ and $N$ are special cases of these when $t=2$.

2. Stolarsky means and Gini means. For the latter use, we recall definitions and some properties of Stolarsky means and Gini means.

The Stolarsky mean $D_{a, b}(x, y)$ of $x>0$ and $y>0(x \neq y)$ of order $(a, b)$ $(a, b \in \mathbb{R})$ is defined as follows:

$$
D_{a, b}(x, y)= \begin{cases}{\left[\frac{b\left(x^{a}-y^{a}\right)}{a\left(x^{b}-y^{b}\right)}\right]^{1 /(a-b)},} & a b(a-b) \neq 0, \\ \exp \left(-\frac{1}{a}+\frac{x^{a} \ln x-y^{a} \ln y}{x^{a}-y^{a}}\right), & a=b \neq 0, \\ {\left[\frac{x^{a}-y^{a}}{a(\ln x-\ln y)}\right]^{1 / a},} & a \neq 0, b=0, \\ \sqrt{x y}, & a=b=0\end{cases}
$$

(see [23]).

The identric, logarithmic, and power means of $x$ and $y$ of order $t$ will be denoted by $I_{t}, L_{t}$, and $A_{t}$, respectively. They are special cases of (2.1). We have $I_{t}=D_{t, t}, L_{t}=D_{t, 0}$, and $A_{t}=D_{2 t, t}$. It is worth mentioning that the Seiffert means $M$ and $N$ can also be expressed in terms of the Stolarsky means. We have

$$
M=D_{2,0}(A, G)=L_{2}(A, G), \quad N=D_{2,2}(A, G)=I_{2}(A, G)
$$

We list below some properties of the Stolarsky means:

(P1) $D_{a, b}(\cdot, \cdot)$ is symmetric in parameters $a$ and $b$, that is, $D_{a, b}(\cdot, \cdot)=D_{b, a}(\cdot, \cdot)$.

(P2) $D_{., .}(x, y)$ is symmetric in variables $x$ and $y$, that is, $D_{, .,}(x, y)=$ D.,. $(y, x)$.

(P3) $D_{a, b}(x, y)$ is a homogeneous function of degree one in its variables, that is, $D_{a, b}(\lambda x, \lambda y)=\lambda D_{a, b}(x, y), \lambda>0$.

(P4) $D_{a, b}(x, y) D_{-a,-b}(x, y)=x y$.

(P5) $D_{a, b}$ increases with the increase in either $a$ or $b$.

(P6) If $a \geq 0$ and $b \geq 0$, then $D_{a, b}$ is logarithmically concave in $a$ and $b$. If $a \leq 0$ and $b \leq 0$, then $D_{a, b}$ is logarithmically convex in $a$ and $b$.

(See $[4,12,23])$. 
In order to state a comparison result for the Stolarsky means, we define the two functions

$$
k(a, b)= \begin{cases}\frac{|a|-|b|}{a-b}, & a \neq b, \\ \operatorname{sign}(a), & a=b,\end{cases}
$$

and, for $a \geq 0$ and $b \geq 0$,

$$
l(a, b)= \begin{cases}L(a, b), & a>0, b>0 \\ 0, & a \cdot b=0 .\end{cases}
$$

The following result is due to Páles [9] and Leach and Sholander [5].

COMPARISON THEOREM. Let $a, b, c, d \in \mathbb{R}$. Then,

$$
D_{a, b}(x, y) \leq D_{c, d}(x, y)
$$

holds true if and only if $a+b \leq c+d$ and

$$
\begin{aligned}
l(a, b) & \leq l(c, d) & & \text { if } 0 \leq \min (a, b, c, d), \\
k(a, b) & \leq k(c, d) & & \text { if } \min (a, b, c, d)<0<\max (a, b, c, d), \\
-l(-a,-b) & \leq-l(-c,-d) & & \text { if } \max (a, b, c, d) \leq 0 .
\end{aligned}
$$

A special case of the Gini mean is

$$
J_{t} \equiv J_{t}(x, y)= \begin{cases}\exp \left(\frac{x^{t} \ln x+y^{t} \ln y}{x^{t}+y^{t}}\right), & t \neq 0, \\ \sqrt{x y}, & t=0\end{cases}
$$

(see [3]). For later, we record the two results

$$
A<J_{1 / 2},
$$

$x \neq y($ see $[10,18])$ and

$$
I_{2 t}^{2}=I_{t} J_{t}, \quad t \in \mathbb{R}
$$

(see [7]). When $t=1$, we write $I$ and $J$ instead of $I_{1}$ and $J_{1}$.

3. Refinements of inequalities (1.5) and (1.7). To this end, we assume that $x \neq y$. We are in a position to prove the following theorem.

THEOREM 3.1. Let $x>0$ and $y>0$. Then,

$$
\begin{aligned}
L & <L(A, G)<M<I(A, G)<\sqrt{\frac{A^{2}+4 A G+G^{2}}{6}}<\frac{A+G}{2} \\
& <\sqrt{\frac{A^{2}+A G+G^{2}}{3}}<N<I .
\end{aligned}
$$


Proof. For the proof of the first inequality in (3.1), we use (2.8) to obtain $\ln A-\ln G<\ln J_{1 / 2}-\ln G$. Application of (2.7) with $t=1 / 2$ gives

$$
\ln A-\ln G<\frac{\sqrt{x} \ln x+\sqrt{y} \ln y}{\sqrt{x}+\sqrt{y}}-\frac{1}{2}(\ln x+\ln y)=\frac{A-G}{L} .
$$

Hence, the assertion follows. The second inequality in (3.1) is an immediate consequence of (P5) and (1.3). The third inequality in (3.1) is a special case of inequality (4.7) (see Theorem 4.2). This can also be established using the Comparison theorem and (2.2). We have $M=D_{2,0}(A, G)<D_{1,1}(A, G)=I(A, G)$. For the proof of the fourth inequality, we employ the following one:

$$
I^{2}(x, y)<\frac{2 A^{2}+G^{2}}{3}=\frac{x^{2}+4 x y+y^{2}}{6}
$$

(see [19]). Replacing $x$ by $A$ and $y$ by $G$, we obtain the desired result. Easy computations show that the fifth, sixth, and seventh terms in (3.1) satisfy the indicated inequalities. The following inequality of Sándor [13]:

$$
I(x, y)>\frac{2 A+G}{3}=\frac{x+\sqrt{x y}+y}{3}
$$

with $x$ replaced by $A^{2}$ and $y$ replaced by $G^{2}$, together with the use of (1.4), completes the proof of the seventh inequality in (3.1). Finally, the last one is established in [22] (see also (1.5)).

Before we state and prove the next result, we recall the definition of the celebrated Gauss arithmetic-geometric mean, denoted by $\operatorname{AGM} \equiv \operatorname{AGM}(x, y)$ and defined as

$$
\mathrm{AGM}=\lim _{n \rightarrow \infty} x_{n}=\lim _{n \rightarrow \infty} y_{n}
$$

where $x_{0}=x, y_{0}=y, x_{n+1}=A\left(x_{n}, y_{n}\right)$, and $y_{n+1}=G\left(x_{n}, y_{n}\right), n=0,1, \ldots$ The importance of this mean is justified by the fact that the complete elliptic integral of the first kind can be numerically evaluated with the aid of the Gauss mean. It is well known that

$$
\begin{aligned}
& \operatorname{AGM}(x, y)=\operatorname{AGM}(A, G), \\
& \operatorname{AGM}(x, y)<\left(\frac{\sqrt{x}+\sqrt{y}}{2}\right)^{2} .
\end{aligned}
$$


THEOREM 3.2. If $x$ and $y$ are positive real numbers, then

$$
\begin{aligned}
L & <L(A, G)<\mathrm{AGM}<\left(\frac{\sqrt{A}+\sqrt{G}}{2}\right)^{2}<P(A, G) \\
& <\frac{A+\sqrt{A G}+G}{3}<I(A, G)<\frac{A+G}{2}<P \\
& <\frac{2 A+G}{3}<I, \\
\frac{1}{P} & <\frac{1}{3}\left(\frac{1}{A}+\frac{4}{A+G}\right)<\frac{1}{3}\left(\frac{1}{A}+\frac{2}{\mathrm{AGM}}\right) .
\end{aligned}
$$

Proof. The first inequality in (3.8) is already established (see (3.1)). The second inequality follows from $L(x, y)<\operatorname{AGM}(x, y)$ (see [2]) and from (3.6), while the third one is an immediate consequence of (3.6) and (3.7). To complete the proof of (3.8), we use the chain of inequalities

$$
\frac{A+G}{2}<P(x, y)<\frac{2 A+G}{3}<I(x, y)
$$

(see $[13,17,21]$ ). Replacing $x$ by $A$ and $y$ by $G$, we obtain inequalities four through six. The seventh inequality in (3.8) follows from $I<A$. The remaining inequalities are those of (3.10). For the proof of (3.9), we use the following result of Sándor [17]:

$$
P^{3}>A\left(\frac{A+G}{2}\right)^{2}
$$

Taking the reciprocals and, next, using the arithmetic mean-geometric mean inequality, we obtain

$$
\frac{1}{P}<\left(\frac{1}{A}\right)^{1 / 3}\left(\frac{2}{A+G}\right)^{2 / 3}<\frac{1}{3} \frac{1}{A}+\frac{2}{3} \frac{2}{A+G}<\frac{1}{3}\left(\frac{1}{A}+\frac{2}{\mathrm{AGM}}\right),
$$

where the last inequality follows from AGM $<(A+G) / 2$ (see, e.g., [14, 25]). The proof is complete.

We close this section with the remark that the logarithmic mean $L$ satisfies an inequality

$$
\frac{1}{L}<\frac{1}{3}\left(\frac{1}{G}+\frac{4}{A+G}\right)<\frac{1}{3}\left(\frac{1}{G}+\frac{2}{\mathrm{AGM}}\right)
$$

This follows from the inequality

$$
L^{3}>G\left(\frac{A+G}{2}\right)^{2}
$$

(see [15]). 
4. Seiffert means of arbitrary order and their inequalities. We begin with the definition of the Seiffert means $M_{t}, N_{t}$, and $P_{t}$ of order $t(t \in \mathbb{R})$. Let $x$ and $y$ be positive real numbers. We define, for $t \neq 0$,

$$
\begin{gathered}
M_{t} \equiv M_{t}(x, y)=\left[L\left(A^{t}, G^{t}\right)\right]^{1 / t}, \\
N_{t} \equiv N_{t}(x, y)=\left[I\left(A^{t}, G^{t}\right)\right]^{1 / t}, \\
P_{t} \equiv P_{t}(x, y)=\left[P\left(A^{t}, G^{t}\right)\right]^{1 / t}, \\
M_{0}=N_{0}=P_{0}=\sqrt{A G} .
\end{gathered}
$$

We note that

$$
\begin{aligned}
M_{t} & =D_{t, 0}(A, G)=L_{t}(A, G), \\
N_{t} & =D_{t, t}(A, G)=I_{t}(A, G) .
\end{aligned}
$$

Proposition 4.1 (duplication formulas). For any $t \in \mathbb{R}$,

$$
\begin{aligned}
& M_{2 t}^{2}=M_{t} A_{t}(A, G), \\
& N_{2 t}^{2}=N_{t} J_{t}(A, G) .
\end{aligned}
$$

Proof. There is nothing to prove when $t=0$. Assume that $t \neq 0$. In order to establish (4.4), we use (1.3) and (1.1) to obtain $M^{2}=L\left(A^{2}, G^{2}\right)=L(A, G) A(A, G)$. Replacing $A$ by $A^{t}$ and $G$ by $G^{t}$ and, next, raising both sides to the power $1 / t$, we obtain the result. For the proof of (4.5), we use (1.4) and (2.9) to obtain $N^{2}=I\left(A^{2}, G^{2}\right)=I(A, G) J(A, G)$. Using the same trick as above, we obtain the asserted result.

To this end, we deal with inequalities satisfied by the Seiffert means of arbitrary order. Our first result reads as follows.

THEOREM 4.2. Let $t \in \mathbb{R}$ and let $\alpha_{k}=(k / n) t(1 \leq k \leq n-1)$ and $\beta_{k}=$ $((2 k-1) / 2 n) t(1 \leq k \leq n)$. If $t>0$, then

$$
\begin{aligned}
\left(\sqrt{N_{t} N_{0}} \prod_{k=1}^{n-1} N_{\alpha_{k}}\right)^{1 / n} & <M_{t}<\left(\prod_{k=1}^{n} N_{\beta_{k}}\right)^{1 / n}, \\
\sqrt{N_{t} N_{0}} & <M_{t}<N_{t / 2}, \\
\sqrt{N_{t} N_{0}} N_{t / 2} & <M_{t}^{2}<N_{t / 4} N_{3 t / 4} .
\end{aligned}
$$

Inequalities (4.6), (4.7), and (4.8) are reversed if $t<0$. 
Proof. A special case of (3.7) in [7] states that, for $a>0$,

$$
\left(\sqrt{I_{a} I_{0}} \prod_{k=1}^{n-1} I_{\alpha_{k}}\right)^{1 / n}<L_{a}<\left(\prod_{k=1}^{n} I_{\beta_{k}}\right)^{1 / n}
$$

with the inequalities reversed if $a<0$. Making use of (4.2) and (4.3), we obtain (4.6). Inequalities (4.7) and (4.8) follow from (4.6) by letting $n=1$ and $n=2$, respectively.

THEOREM 4.3. The following inequalities

$$
\begin{array}{ll}
\frac{N_{t}}{M_{t}}<\left(\frac{N_{1}}{M_{1}}\right)^{2}, & 1<t \leq 2, \\
\frac{N_{t}}{M_{t}}<\frac{L_{t}\left(N_{t}, N_{0}\right)}{N_{0}}, & t>0, \\
M_{t}>L\left(N_{t}, N_{0}\right), & t>0, \\
\frac{M_{t}}{M_{0}}>L_{t}\left(e^{z}, e^{-z}\right), & t>0,
\end{array}
$$

where $z=(A-G) /(A+G)$ are valid. Inequalities (4.11), (4.12), and (4.13) are reversed if $t<0$.

Proof. Function $h(t)=\left[L^{2}\left(x^{t}, y^{t}\right) / I\left(x^{t}, y^{t}\right)\right]^{1 / t}$ is strictly increasing for $t \geq 1$ (see [16]). Replacing $x$ by $A$ and $y$ by $G$, we conclude that

$$
\frac{M_{t}^{2}}{N_{t}}>\frac{M_{1}^{2}}{N_{1}} \text { for } t>1
$$

Hence,

$$
\frac{N_{t}}{M_{t}}<M_{t} \frac{N_{1}}{M_{1}^{2}}
$$

To complete the proof of (4.10), it suffices to show that

$$
M_{t}<N_{1} \text { for } t \leq 2 \text {. }
$$

The third and fourth members in (3.1) give $M_{2}<N_{1}$. Since $M_{t}$ is a strictly increasing function in $t$, we see that (4.16) holds true in the stated domain. For the proof of (4.11), we use the following result:

$$
\frac{I G}{L(I, G)}<L
$$

which is established in [7]. Replacing $x$ by $A^{t}$ and $y$ by $G^{t}$ and, next, raising both sides to the power $1 / t$, we obtain the assertion. 
Inequality (4.12) can be established with the aid of the following one:

$$
D_{t, 0}(x, y)>L\left(I_{t}(x, y), G(x, y)\right) \quad(t>0)
$$

(see [7, (3.12)]). Replacing $x$ by $A$ and $y$ by $G$, we obtain the desired result. For the proof of (4.13), we first prove that

$$
\frac{L_{t}(x, y)}{G(x, y)}>L_{t}\left(e^{z}, e^{-z}\right) \quad(t>0)
$$

where $z=(x-y) /(x+y)$. To this aim, we use the following result:

$$
\frac{1}{b-a} \int_{a}^{b} f(t) d t=\sum_{k=0}^{\infty} \frac{1}{(2 k+1) !}\left(\frac{b-a}{2}\right)^{2 k} f^{(2 k)}\left(\frac{a+b}{2}\right)
$$

(see [8]) with $a=0, b=1$, and $f(t)=x^{t} y^{1-t}(0 \leq t \leq 1)$. Making use of

$$
L(x, y)=\int_{0}^{t} x^{t} y^{1-t} d t
$$

we obtain

$$
L(x, y)=G(x, y) \sum_{k=0}^{\infty} \frac{1}{(2 k+1) !}\left(\frac{\ln x-\ln y}{2}\right)^{2 k}
$$

Hence,

$$
\begin{aligned}
\frac{L\left(x^{t}, y^{t}\right)}{G\left(x^{t}, y^{t}\right)} & =\sum_{k=0}^{\infty} \frac{t^{2 k}}{(2 k+1) !}\left(\frac{\ln x-\ln y}{2}\right)^{2 k} \\
& >\sum_{k=0}^{\infty} \frac{(t z)^{2 k}}{(2 k+1) !}=\frac{\sinh (t z)}{t z} .
\end{aligned}
$$

Here, we have used the arithmetic mean-logarithmic mean inequality $L(x, y)<$ $A(x, y)$. Let $t>0$. Then, (4.23) gives

$$
\frac{L_{t}(x, y)}{G(x, y)}>\left(\frac{\sinh (t z)}{t z}\right)^{1 / t}
$$

On the other hand,

$$
\left(\frac{\sinh (t z)}{t z}\right)^{1 / t}=\left(\frac{e^{t z}-e^{-t z}}{2 t z}\right)^{1 / t}=L_{t}\left(e^{z}, e^{-z}\right)
$$

This completes the proof of (4.19). Inequality (4.13) follows from (4.19) by replacing $x$ by $A$ and $y$ by $G$. The proof is complete. 
Inequalities connecting power means of $A$ and $G$ with generalized Seiffert means are contained in the following theorem.

THEOREM 4.4. Let $t>0$. If $1 / 3 \leq p \leq 2 / 3$ and $q \geq \ln 2$, then

$$
\begin{gathered}
M_{0}^{1 / 3} A_{t / 2}(A, G)^{2 / 3}<M_{t}<A_{p t}(A, G)<N_{t}<A_{q t}(A, G), \\
A_{t}(A, G) M_{t}<A_{2 t / 3}(A, G)^{2}<N_{5 t / 6} N_{7 t / 6}<N_{t}^{2}, \\
A_{t}(A, G) M_{t}<N_{t / 2} N_{3 t / 2}<N_{t}^{2} .
\end{gathered}
$$

Proof. The first inequality in (4.26) follows from (3.14) replacing $x$ by $A^{t}$ and $y$ by $G^{t}$. Remaining inequalities in (4.26) are obtained from

$$
L<A_{1 / 3}<A_{1 / 2}<I<A_{\ln 2}
$$

in a similar way. The first inequality in (4.29) is due to Lin [6], the second one is a consequence of monotonicity of the power mean in its parameter, while the remaining two are established in [11]. See also [24]. It is worth mentioning that (4.29) can be easily established using the Comparison theorem. We omit further details. Inequalities (4.27) and (4.28) follow from

$$
A L<A_{2 / 3}^{2}<I_{5 / 6} I_{7 / 6}<I^{2}
$$

(see [7, (3.9) and (3.7)]) and from

$$
A L<I_{1 / 2} I_{3 / 2}<I^{2}
$$

(see $[7,(3.8)])$, respectively.

In the next theorem, we give bounds on the mean $N_{t}$.

THEOREM 4.5. Let $t>0$. Then,

$$
\left(\frac{2}{e}\right)^{1 / t} A_{t}(A, G)<N_{t}<\left(\frac{4}{e}\right)^{1 / t} A_{t / 2}(A, G) .
$$

Inequalities in (4.32) are reversed if $t<0$.

Proof. A simple calculation shows that (4.32) is a consequence of the inequality

$$
\frac{2}{e} A<I<\frac{2}{e}(A+G) .
$$

The first inequality in (4.33) is due to Alzer [1] and has been rediscovered by Sándor [14]. We now establish the second inequality in (4.33). Let $0<t<1$. The result

$$
(1+t)^{1 / t^{2}}>1+\frac{1}{t}
$$


follows from Bernoulli's inequality. We have $(1+t)^{1 / t^{2}}>1+\left(1 / t^{2}\right) t=1+1 / t$. Dividing both sides by $1+t$, replacing $t$ by $1 / t$, and, next, raising both sides of the resulting inequality to the power 2 , we obtain

$$
\left(1+\frac{1}{t}\right)^{2}>\left(\frac{1}{t^{2}}\right)^{1 /\left(1-t^{2}\right)} .
$$

Putting $t=\sqrt{y / x}$ and multiplying both sides by $y$, we obtain

$$
(\sqrt{x}+\sqrt{y})^{2}>\left(\frac{x^{x}}{y^{y}}\right)^{1 /(x-y)}
$$

which is equivalent to the second inequality in (4.33).

Two inequalities connecting the means $N_{t}$ and $I$ are proven in the following theorem.

THEOREM 4.6. Let $t \geq 2$. Then,

$$
\frac{N_{t}}{I}<\exp \left(\frac{1}{2}-\frac{1}{t}\right)
$$

If $0<t \leq 2$, then

$$
\frac{N_{t}}{I}>\frac{1}{2} \exp \left(1-\frac{1}{t}\right)
$$

Proof. We have

$$
\begin{aligned}
\ln \frac{N_{t}}{G} & =\frac{1}{t} \ln I\left(A^{t}, G^{t}\right)-\ln G=\frac{1}{t}\left[\frac{A^{t} \ln A^{t}-G^{t} \ln G^{t}}{A^{t}-G^{t}}-1\right]-\ln G \\
& =\frac{A^{t}}{A^{t}-G^{t}} \ln \frac{A}{G}-\frac{1}{t}=\frac{A^{t}}{A^{t}-G^{t}} \sum_{k=1}^{\infty} \frac{1}{2 k} z^{2 k}-\frac{1}{t},
\end{aligned}
$$

where $z=(x-y) /(x+y)$. Hence,

$$
\frac{N_{t}}{G}=\exp \left(\frac{A^{t}}{A^{t}-G^{t}} \sum_{k=1}^{\infty} \frac{z^{2 k}}{2 k}-\frac{1}{t}\right) .
$$

Function $A^{t} /\left(A^{t}-G^{t}\right)$ is decreasing in $t$. This in turn implies that $A^{t} /\left(A^{t}-\right.$ $\left.G^{t}\right) \leq A^{2} /\left(A^{2}-G^{2}\right)$ for $t \geq 2$. Since

$$
\frac{A^{2}}{A^{2}-G^{2}}=\left(\frac{x+y}{x-y}\right)^{2}=z^{-2},
$$

(4.40) implies the inequality

$$
\frac{N_{t}}{G} \leq \exp \left(\frac{1}{2}+\sum_{k=1}^{\infty} \frac{z^{2 k}}{2 k+2}-\frac{1}{t}\right) .
$$


Combining this with

$$
\frac{I}{G}=\exp \left(\sum_{k=1}^{\infty} \frac{z^{2 k}}{2 k+1}\right)
$$

(see, e.g., [22]), we obtain

$$
\frac{N_{t}}{I} \leq \exp \left(\frac{1}{2}-\frac{1}{t}+\sum_{k=1}^{\infty}\left(\frac{1}{2 k+2}-\frac{1}{2 k+1}\right) z^{2 k}\right)<\exp \left(\frac{1}{2}-\frac{1}{t}\right)
$$

which completes the proof of (4.37). Assume now that $0<t \leq 2$. Making use of (4.40) and (4.43) and taking into account that $A^{t} /\left(A^{t}-G^{t}\right) \geq A^{2} /\left(A^{2}-G^{2}\right)$, we obtain

$$
\frac{N_{t}}{I} \geq \exp \left(-\frac{1}{t}+U\right)
$$

where $U=1 / 2+\sum_{k=1}^{\infty}(1 /(2 k+2)-1 /(2 k+1)) z^{2 k}$. Since $0<z^{2 k}<1(k \geq 1)$,

$$
U>\frac{1}{2}+\sum_{k=1}^{\infty}\left(\frac{1}{2 k+2}-\frac{1}{2 k+1}\right)=1-\ln 2 .
$$

This, in conjunction with (4.45), gives

$$
\frac{N_{t}}{I}>\exp \left(-\ln 2+1-\frac{1}{t}\right)=\frac{1}{2} \exp \left(1-\frac{1}{t}\right)
$$

The proof is complete.

Seiffert inequalities $N<I$ (see [22, Theorem]) and $N / I>\sqrt{e} / 2$ (see [22, Corollary 2(a)]) follow from (4.37) and (4.38), respectively, by letting $t=2$.

COROLlary 4.7. Let $t<0$. Then,

$$
\frac{N_{t}}{I}<2 \exp \left(-1-\frac{1}{t}\right)
$$

PROof. We use property (P4) and (4.3) to obtain

$$
N_{-t}=\frac{A G}{N_{t}}, \quad t \in \mathbb{R} .
$$

Let $0<t \leq 2$. Application of (4.38) to (4.49) gives

$$
\frac{N_{-t}}{I}<2 \frac{A G}{I^{2}} \exp \left(-1+\frac{1}{t}\right)
$$


Letting $t:=-t$ and using the well-known inequality $A G<I^{2}$, we obtain (4.48) for $-2 \leq t<0$. Since $N_{t}$ is a strictly increasing function in $t$, we conclude that (4.48) holds true for any $t<0$.

We close this section with the following theorem.

THEOREM 4.8. Let $t>0$. Then,

$$
M_{t}<A_{t / 2}(A, G)<P_{t}<\left(\frac{A^{t}+G^{t}+(A G)^{t / 2}}{3}\right)^{1 / t}<N_{t}
$$

Inequalities in (4.51) are reversed if $t<0$.

Proof. We use the inequalities

$$
L(x, y)<\frac{A+G}{2}<P(x, y)<\frac{2 A+G}{3}<I
$$

(see (3.8), Theorem 3.2). Replacing $x$ by $A^{t}$ and $y$ by $G^{t}$, we obtain the desired result.

\section{REFERENCES}

[1] H. Alzer, Aufgabe 987, Elem. Math. 43 (1988), 93 (German).

[2] B. C. Carlson and M. Vuorinen, An inequality of the AGM and the logarithmic mean, SIAM Rev. 33 (1991), 655.

[3] C. Gini, Di una formula comprensiva delle medie, Metron 13 (1938), 3-22 (Italian).

[4] E. B. Leach and M. C. Sholander, Extended mean values, Amer. Math. Monthly 85 (1978), no. 2, 84-90.

[5] _ Multivariable extended mean values, J. Math. Anal. Appl. 104 (1984), no. 2, 390-407.

[6] T.-P. Lin, The power mean and the logarithmic mean, Amer. Math. Monthly 81 (1974), 879-883.

[7] E. Neuman and J. Sándor, Inequalities involving Stolarsky and Gini means, to appear in Math. Pannon.

[8] _ On the Ky Fan inequality and related inequalities, II, in preparation.

[9] Z. Páles, Inequalities for differences of powers, J. Math. Anal. Appl. 131 (1988), no. 1, 271-281.

[10] _ Inequalities for sums of powers, J. Math. Anal. Appl. 131 (1988), no. 1, 265-270.

[11] A. O. Pittenger, Inequalities between arithmetic and logarithmic means, Univ. Beograd. Publ. Elektrotehn. Fak. Ser. Mat. Fiz. (1980), no. 678-715, 15-18.

[12] F. Qi, Logarithmic convexity of extended mean values, Proc. Amer. Math. Soc. 130 (2002), no. 6, 1787-1796, RGMIA Research Report Collection 2 (1999), no. 5, Article 5, 643-652.

[13] J. Sándor, A note on some inequalities for means, Arch. Math. (Basel) 56 (1991), no. $5,471-473$.

[14] _ On certain inequalities for means, J. Math. Anal. Appl. 189 (1995), no. 2, 602-606.

[15] _ On certain inequalities for means. II, J. Math. Anal. Appl. 199 (1996), no. 2, 629-635. 
[16] _ _ On certain subhomogeneous means, Octogon Math. Mag. 8 (2000), 156160.

[17] _ On certain inequalities for means. III, Arch. Math. (Basel) 76 (2001), no. 1, 34-40.

[18] J. Sándor and I. Raşa, Inequalities for certain means in two arguments, Nieuw Arch. Wisk. (4) 15 (1997), no. 1-2, 51-55.

[19] J. Sándor and T. Trif, Some new inequalities for means of two arguments, Int. J. Math. Math. Sci. 25 (2001), no. 8, 525-532.

[20] H.-J. Seiffert, Problem 887, Nieuw Arch. Wisk. (4) 11 (1993), no. 2, 176.

[21]___ Ungleichungen für einen bestimmten Mittelwert [Inequalities for a certain mean value], Nieuw Arch. Wisk. (4) 13 (1995), no. 2, 195-198 (German).

[22] _ Ungleichungen für elementare Mittelwerte [Inequalities for elementary means], Arch. Math. (Basel) 64 (1995), no. 2, 129-131 (German).

[23] K. B. Stolarsky, Generalizations of the logarithmic mean, Math. Mag. 48 (1975), 87-92.

[24] _ The power and generalized logarithmic means, Amer. Math. Monthly 87 (1980), no. 7, 545-548.

[25] M. K. Vamanamurthy and M. Vuorinen, Inequalities for means, J. Math. Anal. Appl. 183 (1994), no. 1, 155-166.

Edward Neuman: Department of Mathematics, Southern Illinois University Carbondale, Carbondale, IL 62901-4408, USA

E-mail address: edneuman@math.siu.edu

József Sándor: Department of Pure Mathematics, Babes-Bolyai University, 3400 ClujNapoca, Romania

E-mail address: jsandor@math . ubbcluj . ro 


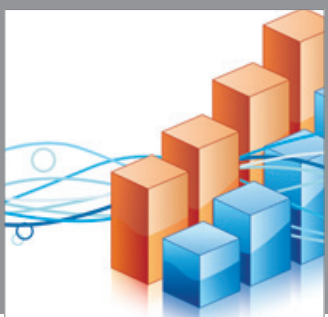

Advances in

Operations Research

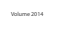

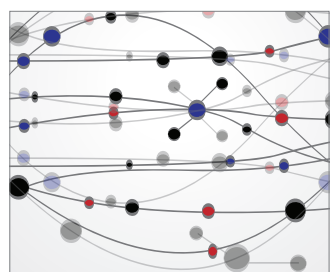

\section{The Scientific} World Journal
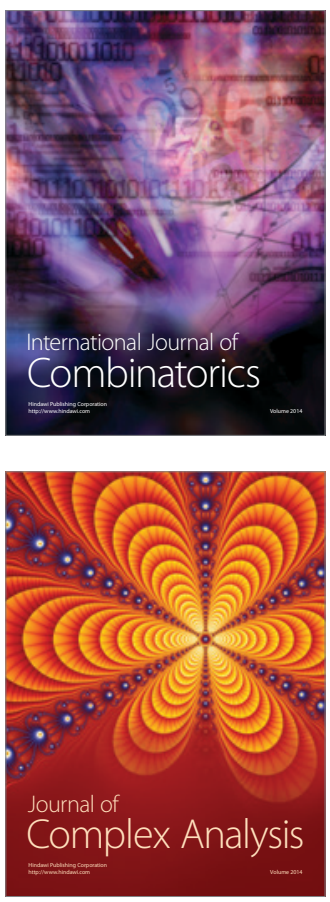

International Journal of

Mathematics and

Mathematical

Sciences
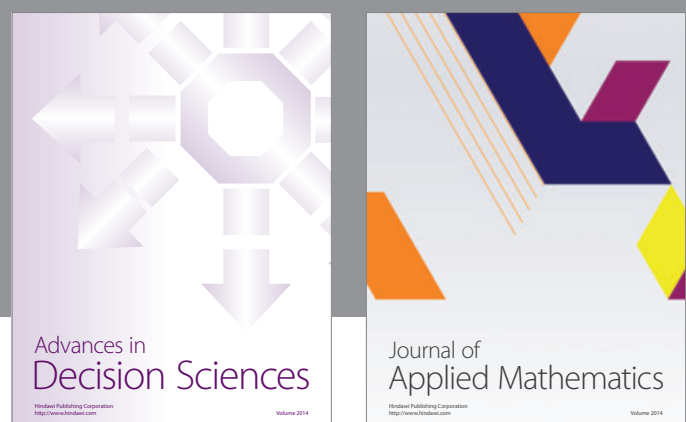

Journal of

Applied Mathematics
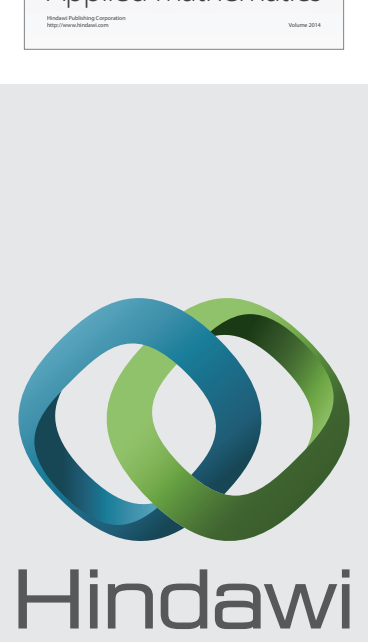

Submit your manuscripts at http://www.hindawi.com
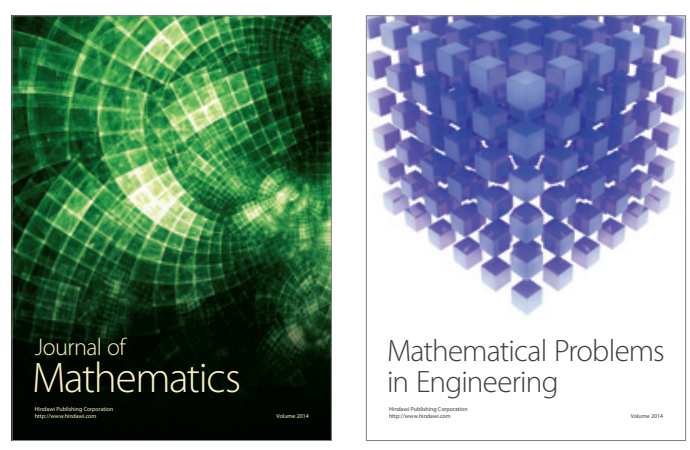

Mathematical Problems in Engineering
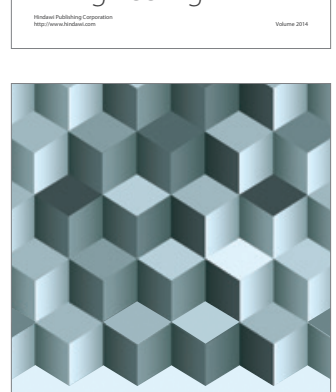

Journal of

Function Spaces
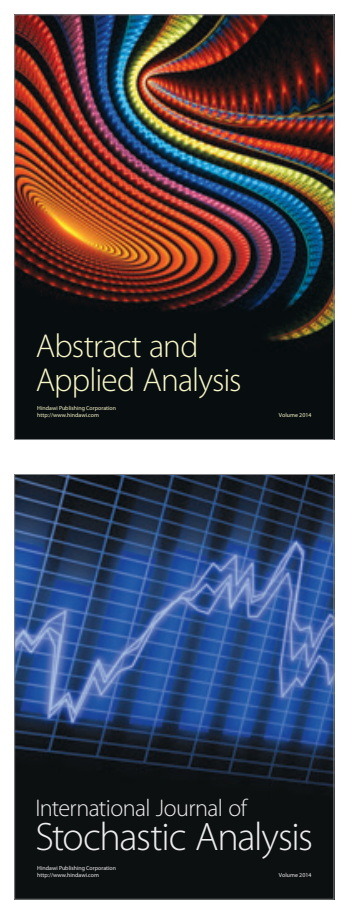

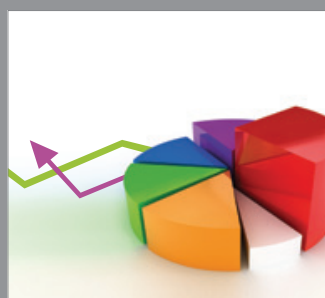

ournal of

Probability and Statistics

Promensencen
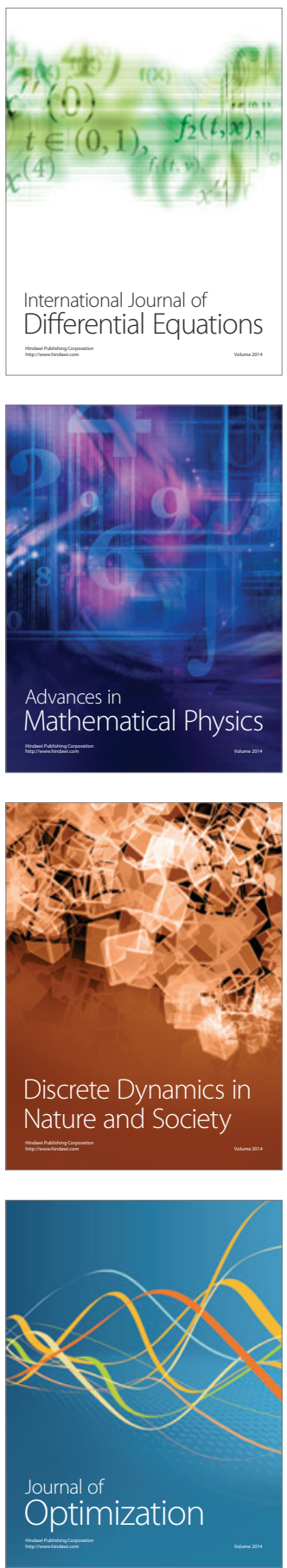